\title{
HER2 Overexpression and Mismatch Repair Deficiency are Correlated with Malignancy in Colorectal Cancer
}

\author{
Hai Luo ${ }^{1, *}$ \\ Lingzhi Cui ${ }^{2, *}$ \\ Kexin Shen ' \\ Ruiqi $\mathrm{Li}^{1}$ \\ Zeming Wang' \\ Zhongshi Xie (D) \\ 'Gastrointestinal Colorectal and Anal \\ Surgery, China-Japan Union Hospital of \\ Jilin University, Changchun, Jilin, People's \\ Republic of China; ${ }^{2}$ General Surgery, The \\ Second Affiliated Hospital of Baotou \\ Medical College, Inner Mongolia \\ University of Science and Technology, \\ Baotou, Inner Mongolia, People's \\ Republic of China \\ *These authors contributed equally to \\ this work
}

Purpose: This study was designed to investigate the correlation between the expression of human epidermal growth factor receptor 2 (HER2), mismatch repair (MMR), and clinicopathological parameters and serum tumor markers in a total of 522 resection samples materials from colorectal cancer (CRC) patients. These data were also used to determine the links between HER2 and MMR expression and prognosis.

Methods: We conducted a retrospective analysis of the clinical data from 522 CRC patients. Immunohistochemistry (IHC) was used to detect HER2 overexpression and MMR deficiency (dMMR) in tumor specimens which were then correlated with various clinicopathological parameters. Prognostic value for HER2 and MMR expression was then evaluated using the data from 105 CRC patients.

Results: HER 2 overexpression was identified in $35.63 \%$ of the samples evaluated in this study, while the total dMMR rate was $12.64 \%$. Expression of HER2 and several, MMR proteins (MLH1, MSH-2, MSH-6, and PMS-2) were then correlated with tumor location. HER2 overexpression is significantly associated with increased depth of tumor invasion, lymph node metastasis, distant metastases, pTNM staging, vascular invasion, nerve infiltration, and serum carcinoembryonic antigen (CEA) levels. HER2 overexpression and dMMR increased with advancing clinical stage. In addition, deficiencies in MLH1 and PMS2 correlated with HER2 overexpression. Finally, the prognostic evaluations revealed that HER2 overexpression was closely associated with poorer clinical outcomes.

Conclusion: HER2 overexpression is significantly correlated with multiple clinicopathological parameters resulting in a poorer prognosis. Moreover, the prognosis of patients with HER2 overexpression was worse, confirming its significance during disease assessment. In clinical practice, clinicians should pay close attention to the HER2 profile of patients as they may require more extensive clinical intervention. In addition, deficiencies in MLH1, MSH-2, MSH-6, or PMS-2 correlate with tumor location, and MLH1 and PMS2 expression is associated with lymph node metastasis and pTNM stage, suggesting that these may be additional markers in CRC risk assessments.

Keywords: human epidermal growth factor receptor 2, mismatch repair deficiency, colorectal cancers, prognosis

\section{Introduction}

Cancer is a significant public health issue worldwide and a leading cause of death. According to 2018 data, colorectal cancer (CRC) was the third most frequently diagnosed cancer, worldwide and ranked second among cancer-related deaths. ${ }^{1}$ Over the past two decades, advances in systemic chemotherapies and targeted therapies,
Correspondence: Zhongshi Xie University, 126 Xian Tai Street, Changchun, Jilin Province, I30033, People's Republic of China Tel +86 I3944I08332

Email surgeonxzs@163.com 
together with complete surgical resection, have significantly improved the disease-free survival (DFS) and overall survival (OS) of CRC patients.

Human epidermal growth factor receptor 2 (HER2) is located on chromosome $17 \mathrm{q} 21$ and is generally found in its inactive state. When stimulated by oncogenic factors, its activated form initiates various signaling pathways enhancing invasion and metastasis in tumor cells., HER2 has been well studied in both breast and gastric cancer, and HER2-targeted therapies are effective in women with breast cancer harboring HER2 overexpression or amplification abnormalities. ${ }^{4,5}$

HER2 overexpression and amplification have also been used as potential therapeutic targets in CRC. ${ }^{6,7}$ Cuyper found that HER2 gene amplification may lead to anti-epidermal growth factor receptor (EGFR)targeted therapy resistance which may serve as a biomarker for predicting anti-EGFR monoclonal resistance. ${ }^{8} \mathrm{~A}$ few studies have reported the incidence rate of HER2 overexpression or amplification in CRC and it varies considerably. ${ }^{9,10}$ This means that studies of HER-2 in colorectal cancer are still underway at various research centers around the world.

Many malignancies are the result of accumulated mutations in various critical genes. ${ }^{11} \mathrm{CRC}$ is thought to develop from one of the two pathways, chromosomal instability (CIN) or microsatellite instability (MSI). ${ }^{12}$ Most CRC arising from CIN is sporadic, with a small number of familial adenomatous polyposis (FAP) developing via this pathway. ${ }^{13}$ MSI results from the mutation or methylation of the mismatch repair genes, with over $90 \%$ of Lynch syndrome and $10 \%$ to $15 \%$ of sporadic colorectal cancer developing via this pathway. ${ }^{14}$ Genetic deficiencies in any one of the genes associated with mismatch repair, MLH-1, MSH-2, MSH-6, and PMS-2, can lead to Lynch syndrome and significantly increase CRC incidence ${ }^{15}$ and previous reports have shown that detection of dMMR can guide both treatment and prognosis in CRC. ${ }^{16}$

This study was designed to explore the relationship between HER2, MLH-1, MSH-2, PMS-2 and MSH-6 expression, and the clinicopathological parameters and serum tumor markers associated with colorectal adenocarcinoma. We also investigated the relationship between HER2 and MMR expression and evaluated the effect of HER2 overexpression and dMMR on CRC prognosis.

\section{Materials and Methods}

\section{Patients and Samples}

This study retrospectively analyzed the clinical data from 522 patients hospitalized between January 2015 and March 2019 at the China-Japan Union Hospital of Jilin University (Jilin, China). The inclusion criteria included: (1) postoperative pathological diagnosis of primary colorectal adenocarcinoma; (2) no preoperative chemoradiotherapy; and (3) complete clinical data. The exclusion criteria were as follows: (1) diagnosis of colorectal carcinoma in situ, squamous cell carcinoma, or adenosquamous cell carcinoma; (2) presence of metastatic colorectal cancer and recurrent malignant tumors; (3) accompanied by other sites and/or other types of primary malignant tumors; (4) serious complications or death within 30 days of surgery. Tumors were staged based on the Tumor-NodeMetastasis (TNM) classification described by the American Joint Committee on Cancer Staging (eighth

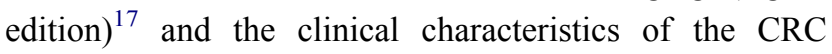
patients recruited to this study are summarized in Table 1.

\section{Immunohistochemical Detection}

Experienced pathologists examined pathological specimens from 522 CRC patients. Samples were embedded in paraffin, sliced into $3 \mu \mathrm{M}$ sections and then dewaxed and washed before incubation in a preheated glass container containing a citric acid buffer solution at $120^{\circ} \mathrm{C}$ for $150 \mathrm{~s}$, to facilitate antigen retrieval). The samples were then left for $30 \mathrm{~min}$ at room temperature and treated with catalase blocker for 20 minutes. Sections were then rinsed with a phosphate buffer solution at $\mathrm{pH} 7.4$ and incubated with primary antibody at $4{ }^{\circ} \mathrm{C}$ overnight. Samples were then rinsed three times using phosphate buffer saline, incubated with secondary antibody and incubate at $37{ }^{\circ} \mathrm{C}$ for 20 minutes. IHC was then developed using diaminobenzidine $(\mathrm{DAB})$ and the slides were counterstained with hematoxylin dehydrated, sealed in resin and then imaged under a microscope. MLH1, MSH2, MSH6, and PMS2 antibodies were purchased from Fuzhou Maixin Biotechnology Development Co., Ltd ...

HER-2 expression in the colorectal cancer tissues was completed as described by the HER-2 detection Guidelines for gastric cancer (2011 edition). ${ }^{18}$ Tissue samples were then evaluated using the HER2 diagnostic criteria for gastric cancer, and scored as follows: $<10 \%$ of tumor cell membrane was HER 2 positive (0); $\geq 10 \%$ tumor cell membrane was weakly or faintly stained, or only part of 
Table I Clinical Characters of CRC Patients Recruited in This Study

\begin{tabular}{|c|c|c|}
\hline Characteristics & Case & Percentage \\
\hline \multicolumn{3}{|l|}{ Gender } \\
\hline Male & 313 & $60.00 \%$ \\
\hline Female & 209 & $40.00 \%$ \\
\hline \multicolumn{3}{|l|}{ Age (year) } \\
\hline$\leq 60$ & 238 & $45.60 \%$ \\
\hline$>60$ & 284 & $54.40 \%$ \\
\hline \multicolumn{3}{|l|}{ Location } \\
\hline Right side colon & 223 & $44.60 \%$ \\
\hline Left side colon & 155 & $29.70 \%$ \\
\hline Rectum & 144 & $25.70 \%$ \\
\hline \multicolumn{3}{|l|}{ T stage } \\
\hline TI & 30 & $5.70 \%$ \\
\hline $\mathrm{T} 2$ & 54 & $10.30 \%$ \\
\hline $\mathrm{T} 3$ & 410 & $78.7 \%$ \\
\hline $\mathrm{T} 4$ & 28 & $5.30 \%$ \\
\hline \multicolumn{3}{|l|}{$\mathrm{N}$ stage } \\
\hline No & 287 & $55.00 \%$ \\
\hline $\mathrm{N}+$ & 235 & $45.00 \%$ \\
\hline \multicolumn{3}{|l|}{ M stage } \\
\hline Mo & 484 & $92.70 \%$ \\
\hline MI & 38 & $7.30 \%$ \\
\hline \multicolumn{3}{|l|}{ Differentiation } \\
\hline Well & 9 & $1.70 \%$ \\
\hline Moderate & 360 & $69.00 \%$ \\
\hline Low & 153 & $29.30 \%$ \\
\hline \multicolumn{3}{|l|}{ Vascular invasion } \\
\hline NO & 381 & $73.00 \%$ \\
\hline YES & $|4|$ & $27.00 \%$ \\
\hline \multicolumn{3}{|l|}{ Nerve infiltration } \\
\hline NO & 396 & $75.90 \%$ \\
\hline YES & 126 & $24.10 \%$ \\
\hline \multicolumn{3}{|l|}{ pTNM stage } \\
\hline 1 & 67 & $12.80 \%$ \\
\hline ॥ & 214 & $41.00 \%$ \\
\hline III & 204 & $39.10 \%$ \\
\hline IV & 37 & $7.10 \%$ \\
\hline \multicolumn{3}{|l|}{ CEA } \\
\hline Neg & 330 & $63.20 \%$ \\
\hline Pos & 192 & $36.80 \%$ \\
\hline \multicolumn{3}{|l|}{ CA19-9 } \\
\hline Neg & 428 & $82.00 \%$ \\
\hline Pos & 94 & $18.00 \%$ \\
\hline \multicolumn{3}{|l|}{ CA242 } \\
\hline Neg & 444 & $85.10 \%$ \\
\hline Pos & 78 & $14.90 \%$ \\
\hline
\end{tabular}

the membrane was HER2 positive $(1+) ; \geq 10 \%$ tumor cells showed weak to moderate basal lateral membrane, lateral membrane, or complete membrane staining $(2+) ; \geq 10 \%$ of tumor cells have intense staining of the basal lateral membrane, lateral membrane, or entire membrane for HER2 $(3+)$.

\section{Detection of Serum Tumor Markers}

Fasting elbow venous blood was submitted to the central research office of the China-Japan Union Hospital of Jilin University for quantitative analysis. Blood samples were centrifuged at $2000 \times \mathrm{g}$ for $5 \mathrm{~min}$ at room temperature and the supernatant was added to the corresponding tumor kit for detection (Luminex Ltd.). All of the laboratory tests were completed using the standard operating procedures and the relevant manufacturer's instructions (Tellegen Corporation) for the tumor kits. The serum cut-off values were as follows: carcinoembryonic antigen (CEA), $5.00 \mathrm{ng} / \mathrm{mL}$; cancer antigen (CA) 19-9, 37.00 U/mL; and cancer antigen (CA) 242, 20.00 $\mathrm{U} / \mathrm{mL}$, any value higher than the cut-off was considered positive.

\section{Follow-Up}

A total of 105 patients were then successfully followed up. In this case, follow-up was defined as any interaction following primary surgical intervention including disease recurrence or death. CRC patients had regular follow-up visits every 3-6 months for the first two years post-surgery. After two years, follow-up was limited to a single annual visit and included CT, colonoscopy, and CEA to allow for the detection of any local recurrence or metachronous disease.

\section{Statistical Analysis}

Correlation between each of the five markers (HER2, MLH1, MSH-2, MSH-6, and PMS-2) and clinicopathological characteristics was analyzed using Pearson's chi-squared $\left(\chi^{2}\right)$ test. Binary logistic regression was applied to evaluate the associations between HER2 and various clinicopathological parameters and correlations among the different markers were assessed using the Spearman correlation. DFS and OS were evaluated using survival analysis techniques (Cox regression, Kaplan-Meier survival analysis, and Log rank tests) with each entry including the date of surgery and follow-up including 1) the most recent follow-up and 2) date of recurrence for DFS or death for OS.

All the statistical analyses were performed using SPSS 25.0 software (SPSS, Chicago, IL) and a P-value of $<0.05$ was considered statistically significant. 


\section{Results}

\section{Clinical Characteristics, HER2 and MMR} Expression in CRC Patients

Of the $522 \mathrm{CRC}$, patient tumor samples 144 presented with CRC of the right-side of the colon, 155 with CRC of the, left side of the colon, and 223 with CRC of the rectum. The cohort comprised 313 men and 209 women, aged between 25 and 89 years, with a median \pm SD age of $61.4 \pm 11.4$ years. HER2 overexpression was detected in 186 cases $(35.63 \%)$ and dMMR in 66 cases $(12.64 \%)$. Of these, 35 cases (6.70\%), 25 cases $(4.79 \%), 20$ cases $(3.83 \%)$, and 52 cases $(9.96 \%)$ were deficient in MLH1, MSH2, MSH6, or PMS2, respectively (Figure 1).

\section{Association Between Each Marker and the Various Clinicopathological}

\section{Parameters}

All five markers mentioned above were investigated for their associations with the various pathological parameters of CRC (Table 2). The expression of each of these five markers correlated with specific tumor locations. HER2 overexpression was associated with the depth of tumor invasion, lymph node metastasis, distant metastases, pTNM staging, vascular invasion, nerve infiltration, and serum CEA level $(\mathrm{P}<0.05)$. However, there were no statistically significant associations between HER2 and gender, age, serum CA19-9, and CA242 levels $(\mathrm{P}>0.05)$. Reduced PMS2 and MLH1 expression was shown to have a significant association with $\mathrm{N}$ stage and pTNM staging. In addition, PMS2 inadequacy was associated with gender and serum CEA levels, while MSH2 was associated with serum CA242 expression (Table 2). To further understand the relationships between HER2 expression and various CRC clinicopathological parameters, we went on to evaluate each relationship using a binary logistic regression analysis. The results of which indicate that increased HER2 expression was significantly associated with lymph node metastasis and pTNM staging when compared to other CRC clinicopathological parameters $(\mathrm{P}<0.05)$ (Table 3).

\section{Correlation of Different Markers Expression}

Among the 52 patients with PMS2 expression deficiency, 33 also exhibited some form of MLH1 inhibition. Of the 22 patients with MSH6 deficiency17 were also shown to present with reduced MSH2 expression. Spearman correlation analysis showed that PMS2 expression correlated with a deficiency of MLH1 (X2=297.408, P $<0.001)$, and MSH6 was associated with a lack of MSH2 (X2=336.213, $\mathrm{P}<0.001$ ). Of the 35 patients with a deficiency in MLH1 25 were also shown to experience an overexpression of HER2. In addition, out of the 52 patients presenting with reduced PMS2 expression, a total of 34 also exhibited increased HER2 expression. Statistical analysis showed that the lack of MHL1 and PMS2 correlated with HER2 overexpression $\quad(\mathrm{X} 2=24.378, \quad \mathrm{P}<0.001 ; \quad \mathrm{X} 2=22.290$, $\mathrm{P}<0.001$, respectively) (Table 4).

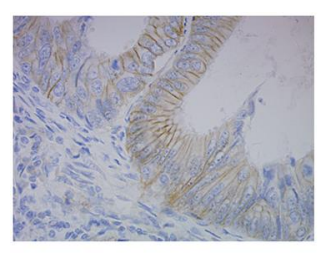

A

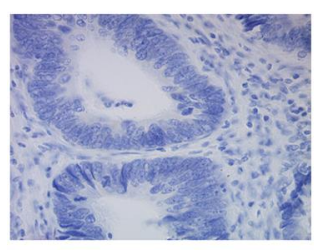

$\mathbf{F}$

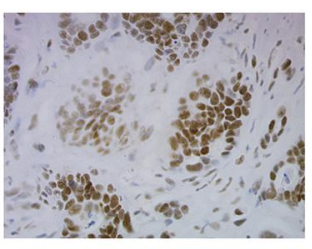

B

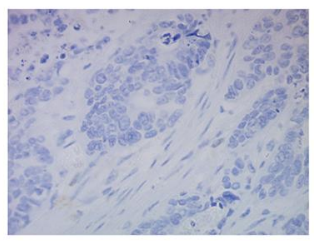

G

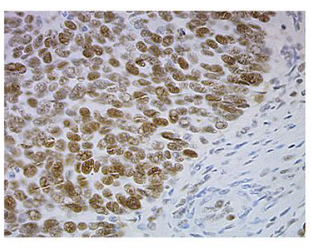

C

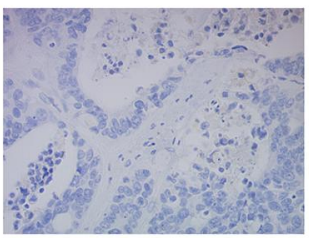

H

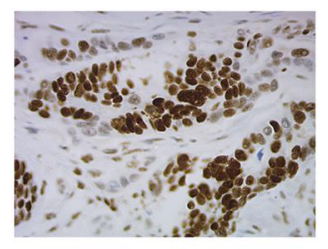

D

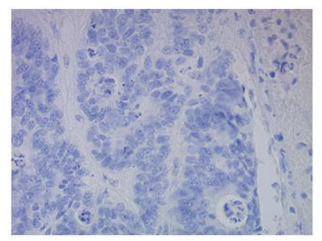

I

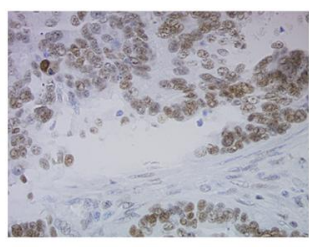

$\mathbf{E}$

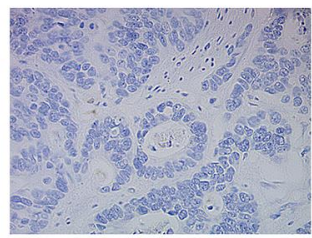

$\mathbf{J}$

Figure I Expression of HER2, MLHI, MSH2, MSH6 and PMS2 markers in CRC tissues (×400). (A) HER2 (+); (B) MLHI (+); (C) MSH2 (+); (D) MSH6 (+); (E) PMS2 (+); (F) HER2 (-); (G) MLHI (-); (H) MSH2 (-); (I) MSH6 (-); (J) PMS2 (-). 


\begin{tabular}{|c|c|c|c|c|c|c|c|c|c|c|}
\hline \multirow{4}{*}{$\frac{\tilde{S}}{\sum_{0}}$} & $a$ & ర్ & ণ্ণ & 范 & 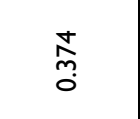 & $\frac{\pi}{0}$ & 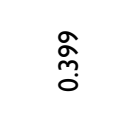 & ্ָ & $\stackrel{\bar{m}}{\stackrel{m}{0}}$ & $\frac{\text { సे }}{0}$ \\
\hline & $x$ & $\begin{array}{l}\text { o } \\
\text { in }\end{array}$ & $\stackrel{\infty}{\stackrel{\circ}{\leftrightarrow}}$ & 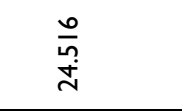 & $\stackrel{\circ}{\stackrel{m}{m}}$ & 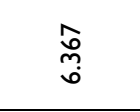 & $\stackrel{\widehat{o}}{-}$ & 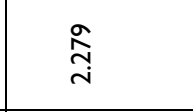 & $\begin{array}{l}\stackrel{2}{0} \\
\text { o. }\end{array}$ & 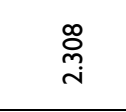 \\
\hline & ŏ & ホั & 윽 윰 & $\underline{\underline{n}} \underset{\mathcal{I}}{\underline{m}}$ & $\stackrel{\infty}{\wedge}$ & సิ ڤ્̊ & $\stackrel{\infty}{\sim} \tilde{m}$ & 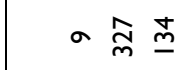 & f̊ำ & $\bar{m}$ 。 \\
\hline & $\stackrel{\substack{0 \\
\mathbf{z}}}{ }$ & sे $\underline{m}$ & $\stackrel{\infty}{\sim} \stackrel{ \pm}{\sim}$ & aิ $\underline{m}$ ㅇ & $\circ \%$ & 요 & +0 & $\circ m a$ & $\stackrel{m}{m}=$ & $\stackrel{m}{m} \simeq$ \\
\hline \multirow{4}{*}{$\begin{array}{l}\frac{0}{5} \\
\frac{1}{5}\end{array}$} & $a$ & 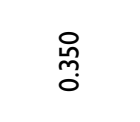 & 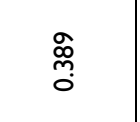 & $\begin{array}{l}\frac{\pi}{0} \\
\dot{0} \\
\mathrm{v}\end{array}$ & ণ্రి & 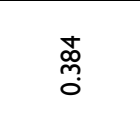 & 恕 & 索 & $\begin{array}{l}\stackrel{0}{\hat{\kappa}} \\
\hat{0}\end{array}$ & 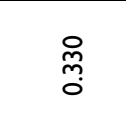 \\
\hline & $x$ & $\underset{\substack{\infty \\
\infty \\
\infty}}{\substack{\infty \\
0}}$ & 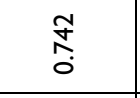 & $\begin{array}{l}\stackrel{\infty}{\leftrightarrow \sim} \\
\stackrel{\sim}{ \pm}\end{array}$ & $\stackrel{\infty}{\stackrel{\infty}{=}}$ & $\stackrel{m}{a}$ & $\bar{m}$ & $\stackrel{\sim}{ \pm}$ & ఫ̊ & \begin{tabular}{l} 
ơ \\
\multirow{2}{\alpha}{} \\
0 \\
\end{tabular} \\
\hline & $\stackrel{\check{0}}{0}$ & مे ڤ્ & $\hat{\text { กิ }}$ & $\overline{\underline{m}} \underline{\underline{n}} \frac{a}{N}$ & $\infty \approx$ & $\stackrel{\sim}{\text { సิ }}$ & $\stackrel{\circ}{+}$ & $\sigma \stackrel{\infty}{m} \stackrel{\wp}{\underline{q}}$ & $\hat{\mathrm{o}} \stackrel{\stackrel{\leftrightarrow}{m}}{\underline{m}}$ & 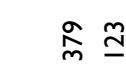 \\
\hline & : & \pm 0 & $=a$ & $\underline{m}+$ & $+\underline{0}$ & \pm 0 & $\underline{\infty} \sim$ & $0 \simeq \infty$ & \pm 0 & $\triangle m$ \\
\hline \multirow{4}{*}{$\frac{\mathfrak{T}}{\frac{5}{5}}$} & $a$ & $\begin{array}{l}\text { to } \\
\stackrel{0}{0}\end{array}$ & 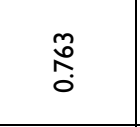 & $\frac{\pi}{0}$ & $\underset{\substack{\alpha \\
\text { o } \\
0}}{0}$ & 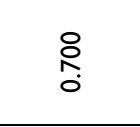 & $\begin{array}{l}\bar{\infty} \\
\stackrel{0}{\circ}\end{array}$ & $\underset{0}{\stackrel{N}{R}}$ & 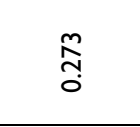 & స్ \\
\hline & $x$ & 음 & 울. & $\begin{array}{l}\hat{\sigma} \\
\stackrel{0}{0}\end{array}$ & $\stackrel{\bar{\infty}}{\stackrel{\sim}{\sim}}$ & $\frac{a}{i}$ & $\frac{\dot{t}}{\dot{\sigma}}$ & $\stackrel{\dddot{\infty}}{\stackrel{\infty}{\infty}}$ & $\stackrel{\infty}{\stackrel{\infty}{్}}$ & $\frac{\mathrm{\sigma}}{0}$ \\
\hline & ¿̊ & $\stackrel{\circ}{\circ} \stackrel{\circ}{\sigma}$ & సั & $\stackrel{\infty}{\simeq} \underline{\underline{n}} \stackrel{\infty}{N}$ & $\infty \frac{1}{7}$ & 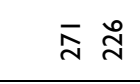 & $\bar{f}$ & $\sigma \underset{m}{*}$ & $\stackrel{m}{\circ}$ & 용 \\
\hline & $\begin{array}{l}\infty \\
\mathbf{z} \\
\mathbf{z}\end{array}$ & 느으 & $\simeq \underline{m}$ & $\underline{0}+$ in & $+\bar{N}$ & $\simeq \sigma$ & $\approx N$ & $0 \mathrm{mo}$ & $\underline{\infty} \wedge$ & $\underline{0}$ \\
\hline \multirow{4}{*}{$\begin{array}{l}\bar{I} \\
\bar{\Sigma}\end{array}$} & $a$ & 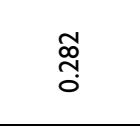 & $\frac{\pi}{i}$ & $\frac{\pi}{\grave{Q}}$ & $\stackrel{\bar{t}}{\dot{\sigma}}$ & $\frac{\pi}{\bar{O}}$ & 兑 & 웅 & $\frac{o}{m}$ & జ̊̊. \\
\hline & $\approx$ & $\stackrel{\infty}{\stackrel{\infty}{!}}$ & $\frac{\grave{m}}{0}$ & $\begin{array}{c}\substack{\infty \\
\stackrel{\infty}{\leftrightarrow} \\
\stackrel{\sim}{\sim}} \\
\end{array}$ & $\underset{\mathrm{d}}{\tilde{\mathrm{d}}}$ & 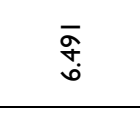 & $\stackrel{\stackrel{\rho}{m}}{=}$ & $\bar{\sigma}$ & $\underline{\text { ọ }}$ & $\stackrel{\stackrel{n}{o}}{\stackrel{p}{m}}$ \\
\hline & 气̆ & $\stackrel{\infty}{\sim} \stackrel{\infty}{\stackrel{\infty}{\sigma}}$ & $\overline{\text { స }}$ & $\underset{\beth}{ \pm} \frac{\infty}{N}$ & $\bar{\infty} \stackrel{\circ}{+}$ & $\stackrel{n}{\sim} \frac{\sigma}{N}$ & 角芦 & $\sigma \hat{m} \bar{I}$ & $\stackrel{\infty}{\mathrm{m}} \stackrel{\text { I }}{ }$ & $\stackrel{+}{m} \stackrel{m}{=}$ \\
\hline & $\stackrel{\infty}{\mathbf{z}}$ & $\pm=$ & $\simeq \stackrel{\infty}{0}$ & $\bar{N} a$ in & $m \approx$ & $\simeq \approx$ & $\bar{m}+$ & $\circ \approx \mathbb{N} \simeq$ & $\tilde{\sim} \simeq$ & $\approx m$ \\
\hline \multirow{4}{*}{$\begin{array}{l}\underset{\tilde{u}}{\Psi} \\
\underset{I}{\mathbf{I}}\end{array}$} & $a$ & $\stackrel{n}{n}$ & $\frac{m}{0}$ & 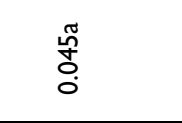 & ฮ̊ర్ & $\frac{\pi}{\grave{Q}}$ & $\frac{\pi}{8}$ & $\frac{\stackrel{m}{0}}{0}$ & $\begin{array}{l}\frac{\pi}{0} \\
\stackrel{0}{0}\end{array}$ & $\frac{\delta}{0}$ \\
\hline & $\approx$ & $\frac{a}{\sigma}$ & 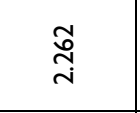 & $\frac{a}{6}$ & $\stackrel{\hat{\oplus}}{\underline{n}}$ & 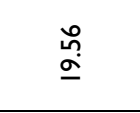 & $\frac{\stackrel{P}{ \pm}}{\dot{ \pm}}$ & 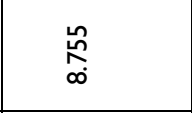 & $\begin{array}{l}\infty \\
\stackrel{0}{ } \\
\dot{ \pm}\end{array}$ & 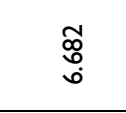 \\
\hline & $\stackrel{0}{0}$ & $\stackrel{n}{=} \pi$ & $n \approx$ & ஸீ గూ & $\underline{\underline{n}} \overline{\underline{I}}$ & $\stackrel{ }{\hat{0}}$ & $\underline{\underline{N}}$ & $\circ \bar{\simeq}$ & 三 & Iิ in \\
\hline & $\begin{array}{l}\infty \\
\mathbf{z} \\
\mathbf{z}\end{array}$ & $\stackrel{\infty}{\stackrel{\infty}{m}}$ & 守 & $\infty \approx \underline{n}$ & oิ & $\stackrel{\infty}{\sim} \stackrel{\infty}{\sim}$ & $\underset{\sim}{ } \pm$ & $\sigma \stackrel{\text { స్ }}{\infty}$ & $\stackrel{\text { N }}{N}$ & సิ \\
\hline 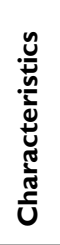 & & 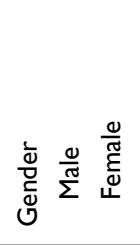 & 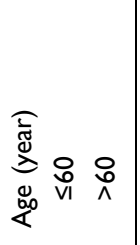 & 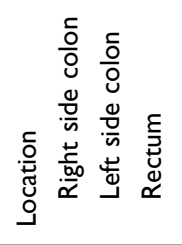 & 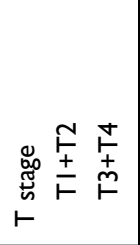 & 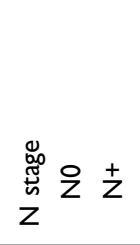 & 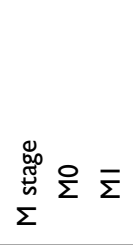 & 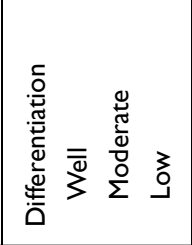 & 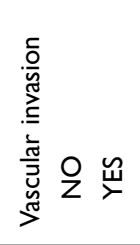 & 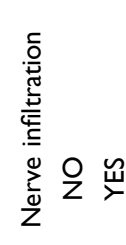 \\
\hline
\end{tabular}




\begin{tabular}{|c|c|c|c|c|c|}
\hline \multirow{4}{*}{$\sum_{0}^{\hat{n}}$} & $a$ & $\begin{array}{l}\frac{\pi}{0} \\
\stackrel{0}{0}\end{array}$ & $\begin{array}{l}\stackrel{\pi}{\tilde{N}} \\
\stackrel{0}{0} \\
0\end{array}$ & $\frac{\hat{0}}{0}$ & $\frac{\nabla}{\circ}$ \\
\hline & $x^{x}$ & $\begin{array}{l}\stackrel{0}{0} \\
\mathbf{0} \\
\underline{0}\end{array}$ & 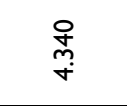 & $\stackrel{\sim}{a}$ & $\stackrel{\text { ָ̊ }}{0}$ \\
\hline & $\grave{0}$ & 뚀 & $\stackrel{+}{0} \stackrel{0}{0}$ & $\stackrel{\circ}{\infty} \bar{\infty}$ & $\bar{q}$ \\
\hline & $\stackrel{\infty}{0}^{\infty}$ & $m \underline{m}$ 는으 & $\stackrel{2}{\sim}$ & gे $\underline{m}$ & Fo \\
\hline \multirow{4}{*}{$\frac{\frac{0}{2}}{\frac{1}{\Sigma}}$} & $a$ & 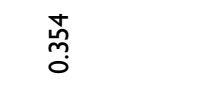 & 恿 & ồ & $\begin{array}{l}\text { Lे } \\
\text { g. }\end{array}$ \\
\hline & $\approx$ & 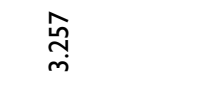 & 蒙 & ڤి & : \\
\hline & $\stackrel{\circ}{0}$ & ఫ & $\frac{\sigma}{m} \stackrel{\infty}{\infty}$ & $\frac{m}{\sigma} \infty$ & $\widehat{\Im}$ \\
\hline & $\stackrel{\infty}{\mathbf{Z}}^{\infty}$ & $m=+N$ & $=a$ & $\underline{n}$ 요 & $\simeq m$ \\
\hline \multirow{4}{*}{$\frac{\mathbf{T}}{\frac{\mathbf{S}}{\Sigma}}$} & $a$ & $\begin{array}{l}\stackrel{\circ}{0} \\
\text { o. } \\
0\end{array}$ & 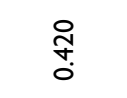 & م̂. & $\frac{\pi}{\frac{\sigma}{0}}$ \\
\hline & $\approx$ & 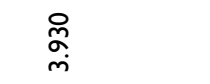 & $\stackrel{\hat{n}}{=}$ & 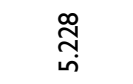 & m̧ \\
\hline & $\stackrel{\circ}{0}$ & ț $\bar{\sim}$ ఏ & $\stackrel{\infty}{m}$ & $\frac{\circ}{\sigma} \hat{\infty}$ & $\stackrel{\sharp}{\mathscr{G}} \Re$ \\
\hline & $\stackrel{\infty}{0}_{\mathbf{z}}^{0}$ & $m \underline{m} \sim \sim$ & $\underline{m} \simeq$ & $\underline{\infty} \sim$ & ㅇ in \\
\hline \multirow{4}{*}{$\frac{\overline{\mathbf{I}}}{\bar{\Sigma}}$} & $a$ & $\frac{\pi}{8}$ & 苂 & $\begin{array}{l}\frac{a}{\sigma} \\
\stackrel{\sigma}{0}\end{array}$ & 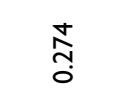 \\
\hline & $x$ & $\begin{array}{l}\stackrel{\underline{D}}{\infty} \\
\stackrel{\leftrightarrow}{\underline{\varphi}}\end{array}$ & $\underset{m}{\bar{m}}$ & $\begin{array}{l}\stackrel{\infty}{0} \\
\text { م̂ }\end{array}$ & $\stackrel{\cong}{\stackrel{Ð}{-}}$ \\
\hline & ถั & $\stackrel{\circ}{\infty} \stackrel{\infty}{\infty} \stackrel{m}{m}$ & $\frac{m}{m} \pm$ & $\bar{q} \bowtie$ & $\frac{N}{\sigma} \mathfrak{n}$ \\
\hline & $\mathbf{z}_{\mathbf{z}}^{\infty 0}$ & $\sim \infty \simeq \infty$ & $\simeq \underline{\infty}$ & $\lambda \infty$ & $\approx m$ \\
\hline \multirow{4}{*}{ 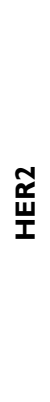 } & $a$ & $\begin{array}{l}\frac{\pi}{8} \\
\dot{0} \\
v\end{array}$ & $\begin{array}{l}\frac{\pi}{Q} \\
\dot{0} \\
\mathrm{v}\end{array}$ & $\begin{array}{l}\text { to } \\
\text { t. }\end{array}$ & $\frac{\omega}{0}$ \\
\hline & $\simeq$ & 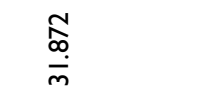 & $\begin{array}{l}\text { ్ㅗ } \\
\stackrel{+}{+}\end{array}$ & R & $\stackrel{\stackrel{\infty}{\stackrel{\infty}{=}}}{=}$ \\
\hline & $\stackrel{0}{0}$ & 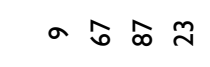 & $\nsubseteq \tilde{\sigma}$ & $\underline{\dot{q}} \hat{m}$ & $\underline{\underline{n}} m$ \\
\hline & $\stackrel{\infty}{z}^{\infty}$ & $\stackrel{\infty}{\mathfrak{4}}$ 三 & $\stackrel{1}{d} 8$ & ๙ิ n & $\overline{\text { న }}$ \\
\hline 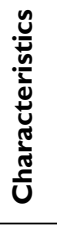 & & 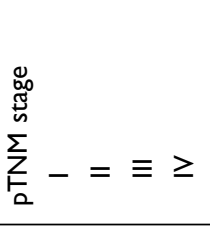 & 虫 & $\frac{\alpha}{\delta} \sum^{\infty}$ & 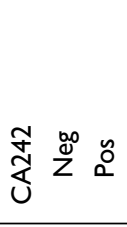 \\
\hline
\end{tabular}


Table 3 Binary Logistic Regression Analysis of HER2 and CRC Clinicopathological Parameters

\begin{tabular}{|c|c|c|c|c|c|c|c|}
\hline & & B & S.E. & Wald & df & $\mathbf{P}$ & $\begin{array}{r}\text { Exp } \\
\text { (B) }\end{array}$ \\
\hline \multirow[t]{6}{*}{ HER2 } & T staging & 0.369 & 0.237 & 2.427 & 1 & 0.119 & 1.446 \\
\hline & $\begin{array}{l}\text { Lymph } \\
\text { node } \\
\text { metastasis }\end{array}$ & 0.964 & 0.478 & 3.455 & 1 & 0.044 & 2.411 \\
\hline & $\begin{array}{l}\text { Distant } \\
\text { metastasis }\end{array}$ & 0.604 & 0.543 & 1.239 & I & 0.226 & 1.830 \\
\hline & $\begin{array}{l}\text { Vascular } \\
\text { Invasion }\end{array}$ & 0.369 & 0.237 & 2.427 & 1 & 0.119 & 1.446 \\
\hline & $\begin{array}{l}\text { Nerve } \\
\text { Infiltration }\end{array}$ & 0.116 & 0.236 & 0.242 & 1 & 0.623 & 1.123 \\
\hline & $\begin{array}{l}\text { PTNM } \\
\text { staging }\end{array}$ & 0.988 & 0.285 & 11.345 & 1 & 0.001 & 2.685 \\
\hline
\end{tabular}

\section{Relationship Between HER2, dMMR and Clinical Stage}

The CRC patients were stratified by clinical stage. I/II/III/ $\mathrm{IV}$, and then the incidence of HER2 overexpression was calculated (Figure 2A). The overexpression rate of HER2 increased with the increasing clinical stage $(\mathrm{P}<0.001)$. This was also true for the dMMR rate (Figure 2B), with our results showing that the $\mathrm{dMMR}$ rates were statistically different between clinical stages $(\mathrm{P}<0.05)$.

\section{Prognostic Value of HER2}

\section{Overexpression and $\mathrm{dMMR}$ in CRC}

A total of 105 patients were followed up in this study, including 55 patients from group 1who were HER2 negative and presented with no MMR deficiency; 38 patients from group 2 who exhibited some HER2 overexpression and no MMR deficiency, and 12 patients from group 3 who were HER2 negative and MMR deficient. The median follow-up time was 26.0 months (range, 2.0-48.0 months). We evaluated DFS and OS in patients who received surgical intervention. The DFS of the three subgroups was $89.1 \%(49 / 55), 65.8 \%(25 / 38)$, and $79.0 \%(9 / 12)$, and the OS of the three subgroups was $92.7 \%$ (51/55), $71.1 \%$ (27/38), and $83.3 \%(10 / 12)$, respectively. The analysis results showed that the prognosis of group 2 was worse than that of the other two subgroups. There were statistically significant differences in DFS $\left(\chi^{2}=6.530, \mathrm{P}=0.010\right.$; $\left.\chi^{2}=4.215, \mathrm{P}=0.041\right)$ and $\mathrm{OS}\left(\chi^{2}=6.380, \mathrm{P}=0.012 ; \chi^{2}=4.010\right.$, $\mathrm{P}=0.044)$ between each of the different groups $(\mathrm{P}<0.05)$ (Figure 3).

These analyses revealed that the prognosis of group 2 was worse than that of the other two subgroups, and the

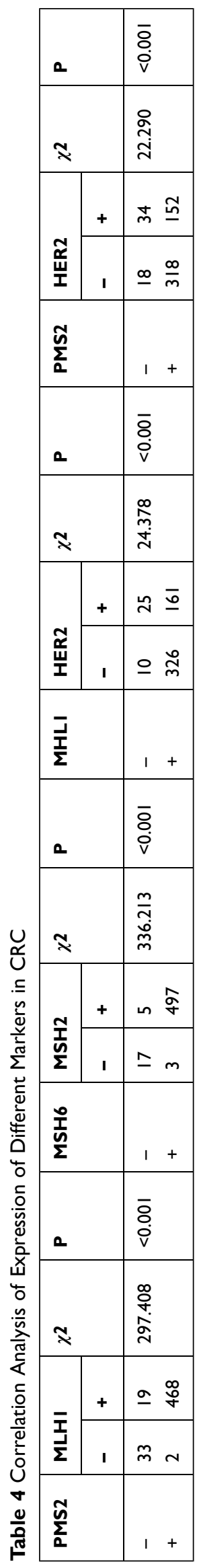


A

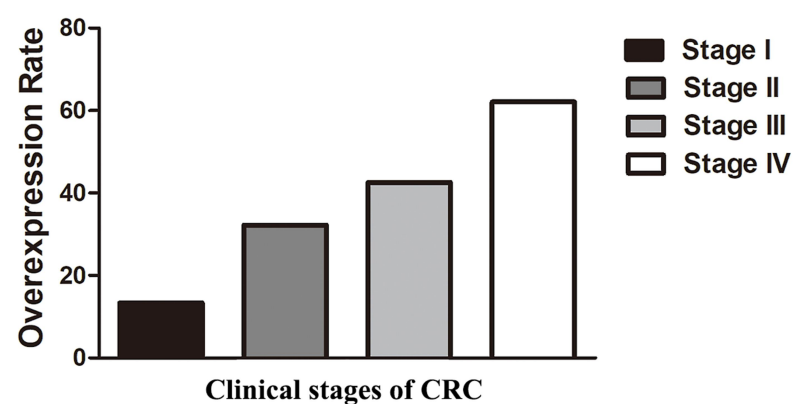

B

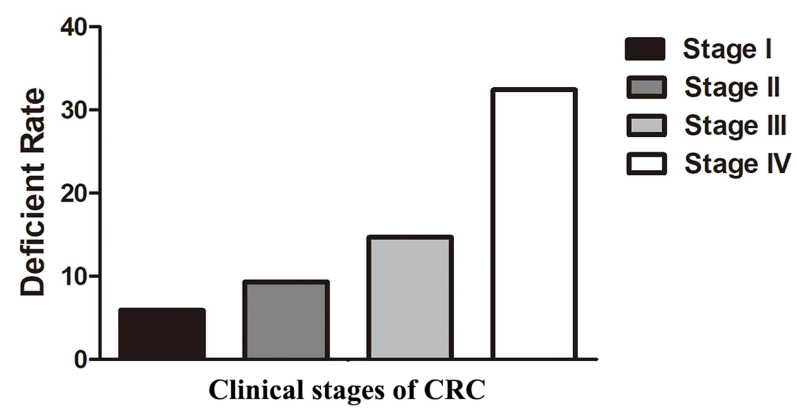

Figure 2 Associations between HER2 and dMMR and clinical stage of CRC. The HER2 overexpression rate (A) and MMR deficient rate (B) increased with increased CRC clinical stages I/II/III/IV. The HER2 overexpression rate and dMMR rate were statistically different in different clinical stages $(P<0.05)$.

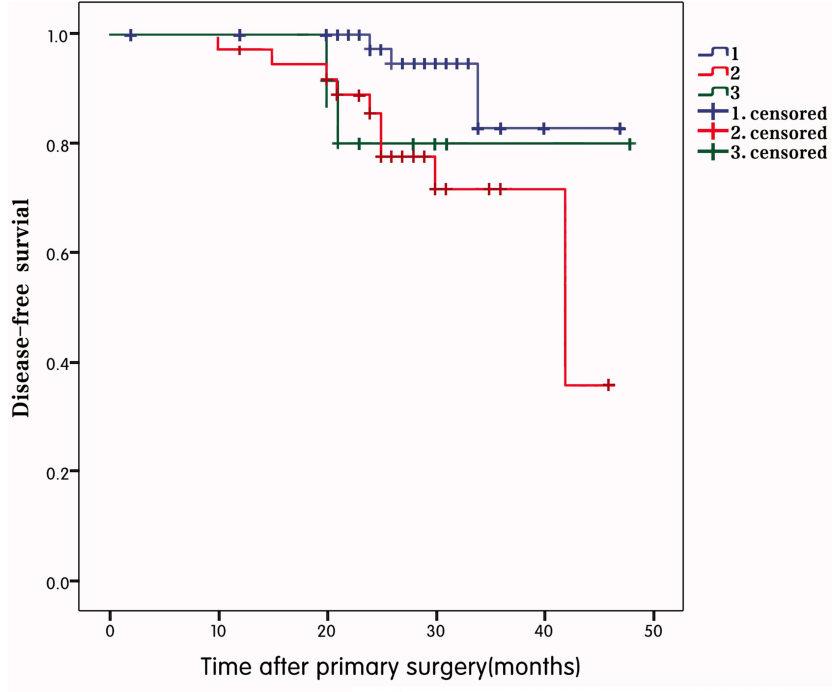

A

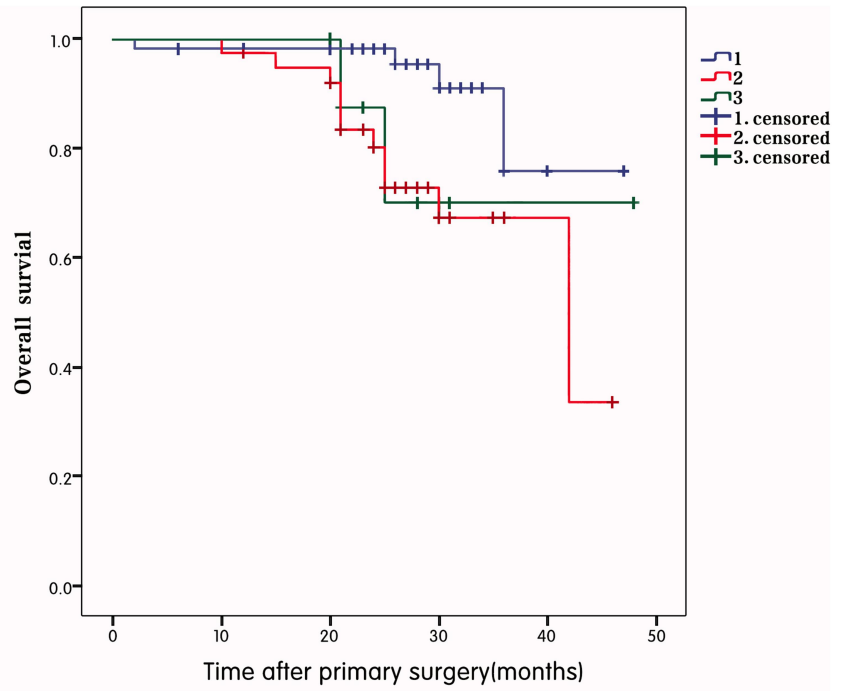

B

Figure 3 Kaplan-Meier survival estimate graphs of disease-free survival (DFS) (A) and overall survival (OS)(B) in different CRC group. I (group I, 55 patients with HER2 negative expression and MMR no deficient), 2 (group 2, 38 patients with HER2 overexpression and MMR no deficient), 3 (group 3,12 patients with HER2 negative expression and MMR deficient).

difference was statistically significant $\quad(\mathrm{P}<0.05)$ (Figure 3).

\section{Discussion}

Our results show that HER2 overexpression correlates with the depth of tumor invasion, lymph node metastasis, distant metastases, pTNM staging, vascular invasion, nerve infiltration, and serum CEA levels. Additionally, HER2 overexpression and dMMR rates increased with the advancing clinical stage. PMS2 and MLH1 deficiency was significantly associated with lymph node metastasis and pTNM staging. Analysis of follow-up results showed that HER2 overexpression may be closely related to prognosis in CRC patients when compared with dMMR.
Reports from the last 20 years have described the overexpression or amplification rate of HER2 in CRC as ranging from between $1.6 \%$ and $81.9 \%{ }^{19-21}$ The ratio of HER2 overexpression in this study was $35.63 \%$ and in contrast to breast and gastric cancers, the diagnostic value of HER2 positivity in CRC has not yet been entirely standardized. The significant differences in HER2 overexpression rates may be related to several factors including, sample size, IHC method, artificial differences in determination methodologies and HER2 evaluation criteria amongst others. Most researchers believe that $\mathrm{IHC}^{+}$, IHC2+, or fluorescence in situ hybridization (FISH) are the good diagnostic criterion for determining HER2 positivity. $^{22,23}$ 
Like HER2, dMMR rates vary between countries and regions. This study used standard IHC to detect MMR expression, and the total deficiency rate was $12.64 \%$, which was similar to previous reports. ${ }^{24}$ In 2017, the National Comprehensive Cancer Network (NCCN) updated its guidelines describing the detection of MMR gene expression and suggesting that this should be performed for all CRC specimens. ${ }^{25}$ Unlike PCR, IHC does not require sophisticated and expensive equipment and is inexpensive and relatively easy to complete. Comparative studies have found that the similarity rate and specificity of the IHC and PCR detection results were as high as over $90 \%$, suggesting that IHC can be used as the primary method for revaluating MMR deficiencies. ${ }^{23}$ It has been suggested that IHC testing compensates for regional genomic differences allowing its greater standardization and facilitating a more uniform evaluation of MMR. This uniformity would make these tests more valuable in clinical settings.

In the current study, we found that HER2 overexpression was related to tumor invasion, lymph node metastasis, distant metastases, vascular invasion, and pTNM staging, but several other studies have failed to show such a relationship. Li reported an association between HER2 expression and tumor size and distant metastases, and Sun meta-analysis showed HER2 amplification was associated with lymph node metastasis and advanced tumor stage. $^{26,27}$ Several studies suggest that HER2 may play some role in tumor progression and would be a valuable prognostic factor for CRC patients. ${ }^{28,29}$

It was also found that patients with HER2 overexpression also had significantly higher favorable rates of serum CEA levels. As we are known, CEA is found in small amounts in the intestinal, pancreatic, and hepatic tissues of average adults. CEA levels are usually high in patients with colorectal adenocarcinoma, and it is a valuable prognostic marker for patients with CRC. ${ }^{30} \mathrm{CEA}$ is positively correlated with HER2, which may be because CEA and HER2 jointly participate in the occurrence, proliferation, and metastasis of CRC tumor cells or the high expression of HER2 stimulates tumor cells to produce more CEA. Does this suggest that these patients have a higher risk of recurrence? At present, there are few relevant studies and mechanism discussions between CEA and HER2, and their relationship needs to be further clarified, which may provide new ideas for the monitoring of CRC metastasis and recurrence.
We found that reduced levels of MLH-1, MSH-2, MSH-6, and PMS-2 all correlated with specific tumor locations, with a deficiency in all four strongly indicating CRC in the right half of the colon which was consistent with the report by Chapusot. ${ }^{31}$ This result may be due to differences in the pathogenesis of left side colon and rectal cancers, or because dMMR in tumor tissues is usually secondary to Lynch syndrome where at least two-thirds of cases are diagnosed in the proximal colon. ${ }^{32-34}$ Karahan et al found that deficiencies in MLH-1 and PMS-2 expression correlated with the development of right colon cancers and positively correlated with low, mucinous differentiation and dense lymphocytic infiltration. ${ }^{11}$ Our study also found that reduced MLH1 and PMS2 expression was associated with several indicators of poor prognosis including local lymph node metastasis and pTNM stage. Interactions between dMMR and changes in the biological behavior of tumor tissues need further investigation.

Based on both the experimental results and Spearman correlation analysis, reduced MLH1 and PMS2, MSH2, and MSH6 protein expression are all interlinked suggesting their co-expression. Several studies have suggested that the working mechanism of MMR protein relies on the production of functional dimers with MLH1 and MSH2 as the dominant proteins and MSH6 and PMS2 as the paired proteins. MLH1 and PMS2 combine to form the heterodimer MutLa, and MSH2 and MSH6 combine to form the heterodimer MutSa. ${ }^{35}$ This study supports the view that when the dominant protein mutates, the heterodimer becomes unstable, leading to a deficiency in the paired protein. Here, we found that some patients did present with a PMS2 deficiency alone. Previous literature reports that a mutation in PMS2 in germline cells results in its inability to localize in the nucleus and results in reduced protein expression, independent of failures in the other proteins in the pathway. ${ }^{36}$

In addition, our results suggest that there is some correlation between MLH1 and PMS2 deficiency and HER2 overexpression with most patients who experience a reduction in MLH1 and PMS2 expression also experiencing significant HER2 overexpression. MMR functions to correct incorrect base insertions during protein repair allowing cells to maintain the accuracy of their gene replication and avoid mutation. When dMMR occurs, it can mediate gene mutation and promote tumors. ${ }^{37}$ Likewise, HER2 can trigger a cascade of multiple critical signaling pathways, including the Ras/Raf/Mek/Erk, the 
Src tyrosine kinase, and the signal transducers and activators of transcription (STAT) pathways. These pathways play an essential role in stimulating tumor cell proliferation, survival, and angiogenesis. ${ }^{8}$ Our results may suggest that dMMR and HER2 overexpressions play synergistic roles during CRC pathogenesis.

The value of HER2 overexpression in both breast and gastric cancer has been confirmed, and numerous targeted drugs have been used to improve therapeutic efficacy in the clinic. However, the role of HER2 in CRC is not well understood and continues to be the subject of significant evaluation. ${ }^{38,39} \mathrm{Lu}$ found that HER2 overexpression is a potential predictive factor for poor outcomes in clinical colorectal cancer, which is supported by the results of this study's longitudinal evaluations. ${ }^{40}$ Similarly, studies have shown that if HER2 expression is regulated by RNA interference in vitro, the proliferation of tumor cells was significantly inhibited, indicating that HER2 is involved in regulating tumor proliferation and may affect the prognosis of patients. ${ }^{41}$ However, a meta-analysis presented a completely different view, suggesting no statistically significant correlation between HER-2 overexpression and prognosis in patients with CRC. ${ }^{19}$ At present, clinical evaluations of the existing anti-HER2 antibodies during CRC treatments suggest that these interventions demonstrate improved efficacy and tolerability. ${ }^{23}$

Reports describing the correlation between MMR and the prognosis of CRC are also controversial. In this study, the prognosis of patients in the dMMR group was not significantly different from that of the regular expression group. Willis suggested that dMMR is a sign of malignant transformation in colorectal adenomas and a risk factor for CRC recurrence. However, Yan reported that dMMR is a sign of good prognosis for patients with stage II and stage III CRC, and these features are significantly different from those with normal MMR genes. ${ }^{42,43}$ There are some exciting studies on the treatment of dMMR and CRC with some studies suggesting that MMR gene deficiency may cause tumor cells to be resistant to chemotherapy drug 5-fluorouracil. Other studies have suggested that irinotecan can improve the 5-year DFS of dMMR patients. ${ }^{44,45}$ We expect other research groups to conduct more research in this area to maximize the benefits to patients.

This study has some limitations. First, the patients were from a single center; and an insufficient sample size may lead to false-positive results. Second, it is not clear whether the preoperative elevation of serum tumor markers affects the results of this study, suggesting that the relationship between HER2, dMMR, and CRC and the mechanism of action underlying these relationships should be further evaluated.

\section{Conclusion}

In summary, HER2 overexpression is closely associated with multiple clinicopathological parameters in CRC patients. Moreover, the clinical prognosis of CRC patients overexpressing HER2 is significantly worse, making this an important parameter during $\mathrm{CRC}$ assessment. In addition, dMMR correlates with tumor location; MLH1 and PMS2 expressions are correlated with lymph node metastasis and pTNM stage, but not correlated with prognosis, which may have some impact on their evaluation during CRC risk assessment.

\section{Ethics Approval and Consent to Participate}

I confirm that I have read the Editorial Policy pages. This study was approved by the Ethics Committee at ChinaJapan Union Hospital part of Jilin University (Jilin, China). This study was conducted in accordance with the Declaration of Helsinki and written informed consent was obtained from all participants.

\section{Disclosure}

The authors have no competing interests to declare.

\section{References}

1. Siegel RL, Miller KD, Jemal A. Cancer statistics, 2015. Cancer J Clin. 2015;65(1):5-29. doi:10.3322/caac.21254

2. Yarden Y, Sliwkowski MX. Untangling the ErbB signalling network. Nat Rev Mol Cell Biol. 2001;2(2):127-137. doi:10.1038/35052073

3. Krishnamurti U, Silverman JF. HER2 in breast cancer: a review and update. Adv Anat Pathol. 2014;21(2):100-107. doi:10.1097/ PAP.0000000000000015

4. Bang YJ, Van Cutsem E, Feyereislova A, et al. Trastuzumab in combination with chemotherapy versus chemotherapy alone for treatment of HER2-positive advanced gastric or gastro-oesophageal junction cancer (ToGA): a Phase 3, open-label, randomised controlled trial. Lancet. 2010;376(9742):687-697; PMID: 20728210. doi: 10.1016/ S0140-6736(10)61121-X

5. Miller K, Cortes J, Hurvitz SA, et al. A randomized Phase 2 trial of MM-302 plus trastuzumab versus chemotherapy of physician's choice plus trastuzumab in patients with previously treated, anthracyclinenaïve, HER2-positive, locally advanced/metastatic breast cancer. BMC Cancer. 2016;16(1):352. PMID: 27259714; PMCID: PMC4893300. doi:10.1186/s12885-016-2385-z.

6. Greally M, Kelly CM, Cercek A. HER2: an emerging target in colorectal cancer. Curr Probl Cancer. 2018;42(6):560-571. doi:10.1016/j. currproblcancer.2018.07.001 
7. Richman SD, Southward K, Chambers P, et al. HER2 overexpression and amplification as a potential therapeutic target in colorectal cancer: analysis of 3256 patients enrolled in the QUASAR, FOCUS and PICCOLO colorectal cancer trials. J Pathol. 2016;238(4):562-570. doi: $10.1002 /$ path.4679

8. De Cuyper A, Van Den Eynde M, Machiels JP. HER2 as a predictive biomarker and treatment target in colorectal cancer. Clin Colorectal Cancer. 2020;19(2):65-72. doi:10.1016/j.clcc.2020.02.007

9. Lee W-S, Park YH, Lee JN, et al. Comparison of HER2 expression between primary colorectal cancer and their corresponding metastases. Cancer Med. 2014;3(3):674-680. doi:10.1002/cam4.228

10. Schuell B, Gruenberger T, Scheithauer W, Zielinski C, Wrba F. HER 2/neu protein expression in colorectal cancer. BMC Cancer. 2006;6 (1):123. doi:10.1186/1471-2407-6-123

11. Karahan B, Argon A, Yıldırım M, Vardar E. Relationship between MLH-1, MSH-2, PMS-2, MSH-6 expression and clinicopathological features in colorectal cancer. Int J Clin Exp Pathol. 2015;8 (4):4044-4053. PMID: 26097592; PMCID: PMC4466979.

12. Kumar V. Robbins and Cotran Pathologic Basis of Disease. Kumar V, Abbas AK, Fausto N, editors. Philadelphia PA: Elsevier Saunders; 2005:269-342.

13. Boland CR. Evolution of the nomenclature for the hereditary colorectal cancer syndromes. Fam Cancer. 2005;4(3):211-218. doi:10.1007/s10689-004-4489-x

14. Delahunty R, Mcmurrick P, Carne P, et al. Screening for mismatch repair deficiency in colorectal cancer[J]. Asia Pac Clin Oncol. 2015;11(S3):52.

15. Ramchander NC, Ryan NA, Crosbie EJ, Evans DG. Homozygous germ-line mutation of the PMS2 mismatch repair gene: a unique case report of constitutional mismatch repair deficiency (CMMRD). BMC Med Genet. 2017;18(1):40. doi:10.1186/s12881-017-0391-x

16. Rex DK, Boland CR, Dominitz JA, et al. Colorectal cancer screening: recommendations for physicians and patients from the U.S. multi-society task force on colorectal cancer. Gastroenterology. 2017;153(1):307-323. doi:10.1053/j.gastro.2017.05.013

17. Amin MB, Edge SB, Greene FL, et al.; Organization of the AJCC Cancer Staging Manual. AJCC Cancer Staging Manual; 2017

18. Editing group for guidelines for the detection of HER2 in gastric cancer. Guidelines for HER2 detection in gastric cancer. Chin J Pathol. 2011;40(8):553-557.

19. Li C, Liu DR, Ye LY, et al. HER-2 overexpression and survival in colorectal cancer: a meta-analysis. J Zhejiang Univ Sci B. 2014;15 (6):582-589. doi:10.1631/jzus.B1300258

20. Tu J, Yu Y, Liu W, Chen S. Significance of human epidermal growth factor receptor 2 expression in colorectal cancer. Exp Ther Med. 2015;9(1):17-24. doi:10.3892/etm.2014.2063

21. Ingold Heppner B, Behrens HM, Balschun K, et al. HER2/neu testing in primary colorectal carcinoma. $B r \quad J$ Cancer. 2014;111 (10):1977-1984. doi:10.1038/bjc.2014.483

22. Seo AN, Kwak Y, Kim DW, et al. HER2 status in colorectal cancer: its clinical significance and the relationship between HER2 gene amplification and expression. PLoS One. 2014;9(5):e98528. doi:10.1371/journal.pone.0098528

23. Sartore-Bianchi A, Trusolino L, Martino C, et al. Dual-targeted therapy with trastuzumab and lapatinib in treatment-refractory, KRAS codon 12/13 wild-type, HER2-positive metastatic colorectal cancer (HERACLES): a proof-of-concept, multicentre, open-label, phase 2 trial. Lancet Oncol. 2016;17(6):738-746. doi:10.1016/ S1470-2045(16)00150-9

24. Ye JX, Liu Y, Qin Y, Zhong HH, Yi WN, Shi XY. KRAS and BRAF gene mutations and DNA mismatch repair status in Chinese colorectal carcinoma patients. World $J$ Gastroenterol. 2015;21 (5):1595-1605. doi:10.3748/wjg.v21.i5.1595
25. Benson AB 3rd, Venook AP, Cederquist L,et al. Colon Cancer, Version 1.2017, NCCN Clinical Practice Guidelines in Oncology. $J$ Natl Compr Canc Netw. 2017;15(3):370-398. doi:10.6004/ jncen.2017.0036

26. Li Q, Wang D, Li J, Chen P. Clinicopathological and prognostic significance of HER-2/neu and VEGF expression in colon carcinomas. BMC Cancer. 2011;11(1):1-6. doi:10.1186/1471-240711-277

27. Sun SJ, Lin Q, Sun Q, et al. High HER-2 protein levels correlate with clinicopathological features in colorectal cancer. J Cancer Res Ther. 2016;12(2):323-333. doi:10.4103/0973-1482.154081

28. Laurentpuig P, Balogoun R, Cayre A, et al. ERBB2 alterations a new prognostic biomarker in stage III colon cancer from a FOLFOX based adjuvant trial (PETACC8). Ann Oncol. 2016;27 (6):4590-4597.

29. Stahler A, Heinemann V, Neumann J, et al. Prevalence and influence on outcome of HER2/neu, HER3 and NRG1 expression in patients with metastatic colorectal cancer. Anticancer Drugs. 2017;28 (7):717-722. doi:10.1097/CAD.0000000000000510

30. McKeown E, Nelson DW, Johnson EK, et al. Current approaches and challenges for monitoring treatment response in colon and rectal cancer. J Cancer. 2014;5(1):31-43. PMID: 24396496. doi:10.7150/ jca.7987

31. Chapusot C, Martin L, Mungra N, et al. Sporadic colorectal cancers with defective mismatch repair display a number of specific morphological characteristics: relationship between the expression of hMLH1 and hMSH2 proteins and clinicopathological features of 273 adenocarcinomas. Histopathology. 2003;43(1):40-47. doi:10.1046/j.1365-2559.2003.01641.x

32. Lynch HT, Snyder CL, Shaw TG, Heinen CD, Hitchins MP. Milestones of Lynch syndrome: 1895-2015. Nat Rev Cancer. 2015;15(3):181-194. doi:10.1038/nrc3878

33. Michailidi C, Papavassiliou AG, Troungos C. DNA repair mechanisms in colorectal carcinogenesis. Curr Mol Med. 2012;12 (3):237-246. doi:10.2174/156652412799218859

34. Mecklin JP. Frequency of hereditary colorectal carcinoma. Gastroenterology. 1987;93(5):1021-1025. doi:10.1016/00165085(87)90565-8

35. Edelbrock MA, Kaliyaperumal S, Williams KJ. Structural, molecular and cellular functions of MSH2 and MSH6 during DNA mismatch repair, damage signaling and other noncanonical activities. Mutat Res. 2013;743-744:53-66. PMID: 23391514; PMCID: PMC3659183. doi:10.1016/j.mrfmmm.2012.12.008

36. van der Klift HM, Mensenkamp AR, Drost M, et al. Comprehensive mutation analysis of PMS2 in a large cohort of probands suspected of lynch syndrome or constitutional mismatch repair deficiency syndrome. Hum Mutat. 2016;37(11):1162-1179; PMID: 27435373. doi: 10.1002/humu. 23052

37. Seifert M, Reichrath J. The role of the human DNA mismatch repair gene hMSH2 in DNA repair, cell cycle control and apoptosis: implications for pathogenesis, progression and therapy of cancer. $J \mathrm{Mol}$ Histol. 2006;37(5-7):301-307. doi:10.1007/s10735-006-9062-5

38. Piccart-Gebhart MJ, Procter M, Leyland-Jones B, et al. Trastuzumab after adjuvant chemotherapy in HER2-positive breast cancer. $N$ Engl $J$ Med. 2005;353(16):1659-1672. doi:10.1056/NEJMoa052306

39. Otsu H, Oki E, Ikawa-Yoshida A, et al. Correlation of HER2 expression with clinicopathological characteristics and prognosis in resectable gastric cancer. Anticancer Res. 2015;35(4):2441-2446. PMID: 25862912.

40. Lu Y, Jingyan G, Baorong S, Peng J, Xu Y, Cai S. Expression of EGFR, Her2 predict lymph node metastasis (LNM)-associated metastasis in colorectal cancer. Cancer Biomark. 2012;11(5):219-226. PMID: 23220854. doi:10.3233/CBM-2012-00282 
41. Ellina MI, Bouris P, Aletras AJ, Theocharis AD, Kletsas D, Karamanos NK. EGFR and HER2 exert distinct roles on colon cancer cell functional properties and expression of matrix macromolecules. Biochim Biophys Acta. 2014;1840(8):2651-2661. PMID: 24792576. doi:10.1016/j.bbagen.2014.04.019

42. Willis JA, Reyes-Uribe L, Chang K, Lipkin SM, Vilar E. Immune activation in mismatch repair-deficient carcinogenesis: more than just mutational rate. Clin Cancer Res. 2020;26(1):11-17. PMID: 31383734; PMCID: PMC6942620. doi:10.1158/1078-0432.CCR-180856

43. Yan WY, Hu J, Xie L, et al. Prediction of biological behavior and prognosis of colorectal cancer patients by tumor MSI/MMR in the Chinese population. Onco Targets Ther. 2016;9:7415-7424. doi:10.2147/OTT.S117089
44. Carethers JM, Chauhan DP, Fink D, et al. Mismatch repair proficiency and in vitro response to 5-fluorouracil. Gastroenterology. 1999;117(1):123-131. PMID: 10381918; PMCID: PMC4343206. doi:10.1016/s0016-5085(99)70558-5

45. Bertagnolli MM, Niedzwiecki D, Compton CC, et al. Microsatellite instability predicts improved response to adjuvant therapy with irinotecan, fluorouracil, and leucovorin in stage III colon cancer: cancer and leukemia Group B protocol 89803. J Clin Oncol. 2009;27 (11):1814-1821; PMID: 19273709; PMCID: PMC2668707. doi: 10.1200/JCO.2008.18.2071

\section{Publish your work in this journal}

Cancer Management and Research is an international, peer-reviewed open access journal focusing on cancer research and the optimal use of preventative and integrated treatment interventions to achieve improved outcomes, enhanced survival and quality of life for the cancer patient.
The manuscript management system is completely online and includes a very quick and fair peer-review system, which is all easy to use. Visit http://www.dovepress.com/testimonials.php to read real quotes from published authors. 